\title{
A 65-year-old man presents with pelvic pain
}

\author{
Ramesh S. Iyer • Puneet Bhargava • \\ Jonathan R. Medverd
}

Published online: 20 April 2010

(C) ISS 2010

Keywords Tophaceous gout · Symphysis pubis

\section{Diagnosis}

Tophaceous gout of the symphysis pubis.

\section{Discussion}

Tophaceous gout of the symphysis pubis is an exceedingly rare event, with only four other cases reported in the literature [1-4]. Gout is the oldest recognized arthropathy and results from the deposition of monosodium urate crystals [5]. Gouty tophi are a consequence of prolonged hyperuricemia and can be found in soft tissues, tendon sheaths, joints, and bony prominences [3]. Calcium may precipitate with the urate crystals to varying degrees, making tophi more apparent on radiographs. Gout most often afflicts the feet, ankles, knees, hands, and elbows, in decreasing order of frequency [5].

The case presentation can be found at doi:10.1007/s00256-010-0898-0

R. S. Iyer

Department of Radiology,

University of Washington Medical Center,

Box 357115, 1959 NE Pacific Street,

Seattle, WA 98195, USA

P. Bhargava $(\bowtie) \cdot J$. R. Medverd

Department of Radiology,

University of Washington \& VA Puget Sound Health Care System,

1660 South Columbian Way,

Seattle, WA 98108, USA

e-mail: bhargp@uw.edu
This case is exceptional because it demonstrates the hallmark imaging characteristics of tophaceous gout in spite of its uncharacteristic location. The symphyseal joint space is preserved. The erosions on either side of the joint are well-demarcated with sclerotic margins. These erosions have been described as "punched-out," resembling "mousebites," with overhanging edges that are typical of gout. The association of these erosions with the hyperdense calcified tophus is a reflection of the indolence of this arthropathy [5]. This case diagnosis may be achieved from the images and information provided.

Following the patient's initial presentation, a skeletal survey and laboratory workup were performed. The serum uric acid level was elevated, in keeping with the suspected diagnosis. Skeletal survey was notable in that the first tarsometatarsal (TMT) joint was unaffected. However, other sites, including the ulnar styloid process, displayed erosions with sclerotic margins and overhanging edges (Fig. 1). The combination of imaging findings, history and laboratory evaluation led to this patient's diagnosis of tophaceous gout involving the symphysis pubis. Synovial fluid analysis of this joint was deferred.

Other conditions that may afflict the symphysis pubis include metastasis, plasmacytoma, primary osseous neoplasm such as chondrosarcoma, septic arthritis, osteoarthritis in isolation or in association with calcium pyrophosphate dihydrate (CPPD) crystal deposition disease, and amyloidosis .

Neoplasms, both primary and secondary, and infection are much less likely given the well-defined pattern of osteolysis with sclerotic margins. Preservation of the joint space and lack of periosteal elevation further mitigate against these diagnoses on imaging alone. Septic joint is unlikely in an afebrile patient with normal white blood cell count. This patient had no systemic symptoms such as weight loss and generalized malaise to suspect malignancy.

Erosions are typical of osteoarthritis involving the symphysis pubis, as in the temporomandibular, acromio- 


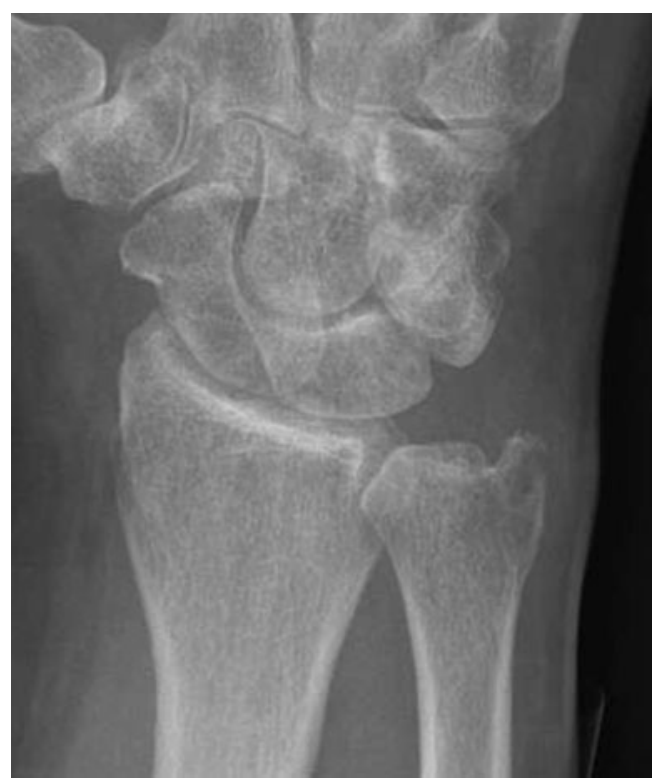

Fig. 1 Oblique radiograph of the right wrist reveals well-demarcated erosions in the ulnar styloid process. There are sclerotic margins proximally and overhanging edges distally

clavicular, and sacroiliac joints [6]. However, association with a calcified soft tissue mass essentially excludes both osteoarthritis and CPPD crystal deposition disease (pseudogout) as the cause of the erosions seen here.

Amyloid arthropathy may present with both soft tissue deposits and erosions with sclerotic margins, along with preservation of the joint space [7]. The history is most helpful in excluding amyloid arthropathy; the patient was not on chronic hemodialysis and did not have a plasma cell dyscrasia. Other, less common causes of amyloid arthropathy include chronic inflammatory disorders, such as rheumatoid arthritis and familial Mediterranean fever [7].

Although not performed in this case, joint aspiration may help elicit a diagnosis. In gout, polarized microscopy of the aspiration fluid reveals negatively birefringent monosodium urate monohydrate crystals [2]. This is in contradistinction to CPPD crystals, which are weakly positively birefringent [8]. Synovial fluid from a septic joint exhibits a very high $(>50,000)$ white blood cell count and may be Gram-stainpositive. Fluid analysis from a joint afflicted with amyloid will stain positive with Congo Red [9].

Tophaceous gout of the symphysis pubis is a rare occurrence, unlikely to be encountered in most musculoskeletal practices. Nonetheless, this case illustrates the classic manifestations of a common arthropathy in an uncommon locale. This condition also highlights the differential considerations for a frequently overlooked joint.

\section{References}

1. Van den Berge M, Vrugt B, Holt C, Smit CJ, Hoogenberg K. Gout as an unusual cause of pelvic pain. Ned Tijdschr Geneeskd. 2006;150(3):151-4.

2. Justiniano ME, Colmegna I, Gimenez CR, Espinoza LR. Tophaceous gout of the symphysis pubis. Arthritis Rheum. 2005;52 (12):4052.

3. Gardner H, McQueen F. Tophaceous gout of the pubic symphysis: an unusual cause of groin pain. Ann Rheum Dis. 2004;63(7):767-8.

4. Castagnoli M, Boileau De Paulis L. Case of gouty pubic symphysis syndrome. Clin Ter. 1975;72(1):81-8.

5. Brower AC. Arthritis in black and white. 2nd ed. Philadelphia: Saunders; 1997. p. 325-41.

6. Helms CA. Fundamentals of skeletal radiology. 3rd ed. Philadelphia: Elsevier Saunders; 2005. p. 113-40.

7. Sheldon PJ, Forrester DM, Learch TJ. Imaging of intraarticular masses. Radiographics. 2005;25(1):105-19.

8. Turgeon ML. Clinical hematology: theory and procedures. 4th ed. Philadelphia: Lippincott, Williams and Wilkins; 2005. p. 424.

9. Lakhanpal S, Li CY, Gertz MA, Kyle RA, Hunder GG. Synovial fluid analysis for diagnosis of amyloid arthropathy. Arthritis Rheum. 1987;30(4):419-23. 